\title{
ON COMPACT MULTIPLIERS OF BANACH ALGEBRAS
}

\author{
HERBERT KAMOWITZ
}

\begin{abstract}
We show that if the maximal ideal space of a commutative semisimple Banach algebra $B$ contains no isolated points, then every compact multiplier is trivial.
\end{abstract}

In [1] and [2] it was shown that if a commutative semisimple Banach algebra $B$ satisfies certain regularity conditions and if the maximal ideal space of $B$ contains no isolated points, then every compact multiplier of $B$ is trivial. ( $T$ is a multiplier of $B$ if $T$ is a linear operator satisfying $T(f g)=f \cdot T g$ for $f, g \in B$.) In this note we show that the regularity conditions used in [1] and [2] are unnecessary. Specifically we prove the following.

ThEOREM. Let $B$ be a commutative semisimple Banach algebra and $T$ a compact multiplier of $B$. If the maximal ideal space of $B$ contains no isolated points, then $T=0$.

Proof. Let $X$ denote the maximal ideal space of $B$ and assume that $X$ contains no isolated points. Since $T$ is a multiplier of $B$, there exists a complex-valued continuous function $u$ on $X$ with $(T f)^{\wedge}(x)=u(x) \hat{f}(x)$ for all $f \in B$ and $x \in X$. We will show first that for each $x \in X, u(x)$ is an eigenvalue of the adjoint $T^{*}$ of $T$. Indeed, for $x \in X$, let $e_{x}$ denote the linear functional in $B^{*}$ which is evaluation at $x$, i.e. $e_{x}(f)=\hat{f}(x)$. Then for each $f \in B$, we have $\left(T^{*} e_{x}\right)(f)=e_{x}(T f)=(T f)^{\wedge}(x)=$ $u(x) \hat{f}(x)=u(x) e_{x}(f)$. Thus $T^{*} e_{x}=u(x) e_{x}$ which proves that $u(x)$ is an eigenvalue of $T^{*}$.

Now $T$, and hence $T^{*}$, is compact, so that the spectrum of $T^{*}, \sigma\left(T^{*}\right)$, is a denumerable set with 0 as its only possible limit point. $\sigma\left(T^{*}\right)$ also has the property that every nonzero element in $\sigma\left(T^{*}\right)$ is an eigenvalue of finite multiplicity. Suppose $x_{0}$ is a point in $X$ which is not an isolated point. We claim that $u\left(x_{0}\right)=0$. Indeed, suppose $u\left(x_{0}\right) \neq 0$. Since $u$ is a continuous function on $X$ and $x_{0}$ is a limit point of $X$, for each positive integer $n$, there exists an element $x_{n}, x_{0} \neq x_{n} \in X$, with $\left|u\left(x_{n}\right)-\left(x_{0}\right)\right|<1 / n$. However, each nonzero eigenvalue of $T^{*}$ has finite multiplicity and so $u\left(x_{n}\right)=u\left(x_{0}\right)$ for only finitely many $n$. Therefore $u\left(x_{0}\right)$ is a limit point of $\left\{u\left(x_{n}\right)\right\} \subset \sigma\left(T^{*}\right)$. However 0 is the only possible limit point of $\sigma\left(T^{*}\right)$ since $T^{*}$ is compact. This contradiction shows that $u\left(x_{0}\right)=0$. Since no element in $X$ is an isolated point, by hypothesis, we conclude that $u(x)=0$ for all $x \in X$. Hence

Received by the editors November 9, 1979.

1980 Mathematics Subject Classification. Primary 47B37; Secondary 46H05. 
$(T f)^{\wedge}(x)=u(x) \hat{f}(x)=0$ for all $x \in X$ and $f \in B$. Therefore $(T f)^{\wedge}=0$ for all $f \in B$, and since $B$ is semisimple, $T f=0$ for all $f \in B$, as claimed.

We remark that if the maximal ideal space $X$ of $B$ has isolated points, then there exist nonzero compact multipliers of $B$. For, if $x_{0}$ is an isolated point of $X$, then by Silov's Idempotent Theorem, there is an idempotent $E$ in $B$ satisfying $\hat{E}(x)=1$ if and only if $x=x_{0}$. Then the operator $T$ defined by $T f=E f=\hat{f}\left(x_{0}\right) E$ is clearly a nonzero multiplier which is compact since its range is one-dimensional.

Finally we remark that if $H$ denotes the Hilbert space of square summable sequences with component-wise multiplication, then $H$ is a commutative semisimple Banach algebra with discrete maximal ideal space. If we let $\left\{a_{n}\right\}$ be a sequence of complex numbers converging to 0 , then the operator $T:\left\{x_{n}\right\} \rightarrow\left\{a_{n} x_{n}\right\}$ is a nonzero compact multiplier of $H$ and $\sigma(T)=\left\{a_{n} \mid n\right.$ is a positive integer $\} \cup\{0\}$.

\section{BIBLIOGRAPHY}

1. M. Dutta and B. Tewari, On multipliers of Segal algebras, Proc. Amer. Math. Soc. 72 (1978), 121-124.

2. S. H. Friedberg, Compact multipliers on Banach algebras, Proc. Amer. Math. Soc. 77 (1979), 210.

Department of Mathematics, Universtty of Massachusetts at Boston, Dorchester, MasSACHUSETTS 02125 